\title{
ESTRATEGIAS DE AFRONTAMIENTO AL ESTRÉS EN UNA MUESTRA DE JÓVENES UNIVERSITARIOS URUGUAYOS
}

\section{COPING STRATEGIES IN A SAMPLE OF URUGUAYAN UNIVERSITY STUDENTS}

\author{
Laura Mariana Scafarelli Tarabal \\ Universidad Católica del Uruguay \\ Reina Coral García Pérez \\ Universidad Católica del Uruguay
}

\begin{abstract}
Resumen: El estudiante universitario, especialmente en su primer año de formación, se enfrenta a diversos cambios, transiciones y dificultades distintas a las que vivió hasta el momento, y, generalmente, se debate entre afrontarlas como el adolescente que está dejando de ser o como el adulto que aún no es. El afrontamiento atraviesa toda su vida; no sólo en lo académico sino también en lo emocional, familiar, vincular y social. Este estudio tiene como objetivos determinar si el perfil de afrontamiento de los estudiantes universitarios de cinco carreras diferentes varía de acuerdo a la carrera que cursan, por un lado, y según el sexo por otro. La presente investigación, de tipo exploratoria, descriptiva y comparativa, se realizó mediante la administración de la escala ACS a 139 estudiantes de Derecho, Educación Inicial, Ingeniería, Odontología y Psicología de primer año de Universidad. Se hallaron diferencias significativas según carrera y sexo.
\end{abstract}

Palabras clave: Afrontamiento, Estrés, Diferencias de sexo.

\begin{abstract}
University students, especially freshmen, are faced with a variety of changes, transitions and difficulties, different from those they are used to, and are generally, are faced with the challenge of coping with them either as the teenagers they still are or as the adults they are becoming. The changes are wholesome and happen emotionally, socially, in the family, and in their relationships. The purpose of this study is to determine if the coping profile of college students in five different majors varies according to their major, and if sex differences are shown. This exploratory, descriptive and comparative research used the ACS scale on 139 Law, Early Childhood Education, Engineering, Odontology, and Psychology Freshmen Students at the University. Significant differences were found in the variables major and sex.
\end{abstract}

Keywords: Coping, Stress, Sex Differences.

\section{INTRODUCCIÓN}

La sociedad contemporánea está caracterizada por un alto nivel de exigencias y una alta competitividad, lo cual nos enfrenta a diversas situaciones que nos demandan el desarrollo de procesos adaptativos. Desde el enfoque transaccional del estrés, Lazarus y Folkman (1987) postulan que el estrés se desarrolla cuando el individuo percibe de forma subjetiva un desequilibrio entre las demandas que se le hacen y los recursos con los que cuenta para manejar dichas demandas. Lo que significa una demanda desmesurada para una persona puede que no lo sea para otra debido a que todos tenemos vulnerabilidades distintas frente al estés. Incluso, puede que una persona sea más o menos vulnerable al estrés en distintos momentos de su vida o frente a diversas situaciones (Lazarus y Folkman, 1987; Lazarus y Lazarus, 1994; Lazarus, 1999).

Varios autores concuerdan que cierta cantidad de estrés es necesaria para proveer la energía requerida para mantenerse con vida, conseguir las metas, comprometerse en relaciones y adaptarse a las influencias externas constantemente cambiantes (Lazarus y Lazarus, 
1994; Johnson y Johnson 2002). Además, el estrés no sólo es inevitable y necesario, sino que en muchas situaciones es ampliamente deseable. Las personas buscan experimentar ciertos tipos de estrés y lo disfrutan, por ejemplo, al practicar deportes de alto riesgo. Para diferenciar este tipo de estrés necesario y/o deseable de las situaciones inesperadas en las que las personas se enfrentan a la desventura o la calamidad, Johnson y Johnson (2002) utilizan el término "adversidad" para referirse a estas últimas. Algunas situaciones de adversidad serían: el rechazo de otros, la muerte de un ser querido o una enfermedad grave.

El afrontamiento al estrés implica un conjunto de acciones cognitivas y afectivas que se manifiestan en respuesta a una preocupación particular y constituyen un intento para devolver el equilibrio o suprimir la perturbación. Esto puede hacerse solucionando el problema, o sea, removiendo el estímulo, o acomodándose a la preocupación sin encontrar una solución al problema (Frydenberg y Lewis, 1993; Lazarus, 1999).

El afrontamiento son manifestaciones cognitivas y conductuales que se ponen en marcha como un intento de regular el estrés y las emociones asociadas a él, más allá de si ese intento es exitoso o no (Lazarus y Lazarus, 1994). El afrontamiento moldea nuestras emociones y su función más importante es regularlas una vez generadas; así como resolver los problemas que las han provocado.

Johnson y Johnson (2002) consideran que para desarrollarse de forma saludable y vivir de modo productivo y feliz, los jóvenes necesitan aprender cómo afrontar constructivamente la adversidad y el estrés. Los jóvenes universitarios, especialmente al inicio de sus carreras, se ven expuestos a diversas situaciones estresantes derivadas de la elección de nuevos planes de estudio e integración a nuevos ámbitos académicos.

Desde esta perspectiva de los fenómenos de estrés y afrontamiento, nos interesa especialmente el estudio de las formas o modos de afrontar que tienen los estudiantes universitarios que cursan distintas carreras.

"La forma de afrontar los problemas puede tenerse en cuenta cuando se trata de establecer planes de carrera puesto que al examinar el repertorio de conductas de afrontamiento de un sujeto éstas pueden ser más adecuadas para ciertos campos profesionales que para otros. Por ejemplo, en profesiones que implican contacto personal las estrategias relacionadas con el apoyo social pueden ser de gran ayuda, mientras que en las carreras científicas el uso excesivo de la fantasía puede ser perturbador" (Frydenberg y Lewis, 1996a; 15).

Frydenberg (1997), una de las autoras de la escala que administramos en este estudio, desarrolla la teoría propuesta por Lazarus y elabora un nuevo modelo de afrontamiento, en el cual enfatiza la reevaluación del resultado. Esta autora postula que luego de una valoración de la situación, el individuo estima el impacto del estrés, esto es, si las consecuencias llevarán más probablemente a la pérdida, al daño, a la amenaza o al desafío y qué recursos (personales o interpersonales) tiene a su disposición para manejarlo. Luego de la respuesta, el resultado es reevaluado (terciariamente valorado o revalorado), y en base a ello puede darse otra respuesta. De este modo, se da un mecanismo de retroalimentación, el cual determina si las estrategias empleadas serán utilizadas nuevamente generando un desarrollo en el repertorio de afrontamiento o serán descartadas como futuras estrategias, según su empleo sea evaluado como eficaz o no de acuerdo con el juicio del propio individuo.

En suma, el afrontamiento puede ser considerado como un proceso multidimensional, una serie de eventos que continúan durante toda la vida como resultado del interjuego de diversos determinantes. Esencialmente, es una interacción dinámica entre las personas y su ambiente (Frydenberg, 2002).

Bajo esta perspectiva, Erica Frydenberg y Ramón Lewis realizaron investigaciones durante 5 años con el objetivo de construir una escala para medir los modos o formas de afrontar que informan preferir los adolescentes cuando se encuentran en dificultades: la Adolescent Coping Scale (ACS). Esta escala fue publicada en 1993 adaptada y baremada al español por Pereña y Seisdedos en 1996 (Frydenberg y Lewis, 1996) y adaptada para Uruguay por García Pérez en 2008. Esta escala consta de dos formas; una general y otra específica. 
Utilizando esta escala se han encontrado diferencias significativas en cuanto a las estrategias de afrontamiento que emplean estudiantes de distintas carreras universitarias (Frydenberg y Rowley, 1998). Estos autores administraron el ACS en su forma específica y hallaron diferencias significativas en el uso de las estrategias: buscar apoyo social, distracción física, buscar ayuda profesional, invertir en amigos íntimos, acción social y reducción de la tensión entre los estudiantes de: Medicina, Psicología y Educación. En todos los casos los estudiantes de Psicología tuvieron la puntuación media más alta (y con diferencia significativa) indicando el nivel más alto de uso de las estrategias anteriormente mencionadas.

Frydenberg y Lewis (1991) señalan que las mujeres buscan más apoyo social y tienden a focalizarse en las relaciones más que los varones. Esto sugiere que ellas aceptan más la comprensión y el apoyo, expresan sus sentimientos y comunican a otros lo que les sucede en mayor medida que los varones. Además, las mujeres emplean más estrategias relacionadas con esperar que ocurra lo mejor y hacerse ilusiones; por ejemplo, fantasear, soñar despiertas y desear que suceda un milagro. En este sentido, aunque el estereotipo de las mujeres que esperan a que "el príncipe encantado acuda a su rescate en un caballo blanco" no refleja exactamente el pensamiento de las chicas en la actualidad aún permanecen ciertas fantasías al respecto. Otra hipótesis que explicaría que las mujeres empleen con mayor frecuencia la estrategia de hacerse ilusiones frente a un problema podría ser que se perciben a sí mismas con cierta carencia de fortaleza, por lo cual la "expectación" se toma como un sustituto para la acción. A pesar de que las mujeres se hacen más ilusiones que los varones, usan estrategias de afrontamiento centradas en el problema en la misma medida que ellos. Esto indica que aunque están deseando que suceda lo mejor no fallan en tomar acciones para manejar sus problemas (Frydenberg y Lewis, 1991).

Frydenberg y Lewis (1993) señalan que un mayor uso de la reducción de la tensión por las mujeres también puede ser interpretado como una falta de fortaleza percibida, de modo que estrategias como llorar, gritar, tomar alcohol o drogas las ayude a alivianar el estrés cotidiano. Por otra parte, los varones reportan usar más la distracción física que las mujeres.
Frydenberg y Lewis (2000) indican que las mujeres se perciben a sí mismas como carentes de estrategias de afrontamiento en mayor medida que los varones. Si bien esta reflexión podría hacernos pensar que los varones son más capaces de seguir con sus vidas a pesar de los problemas empleando estrategias como buscar diversiones relajantes y mantenerse en forma; el hecho de que los varones tiendan más a ignorar los problemas y reservarlos para sí podría estar demostrando negación de su parte y esto podría tener un costo elevado (Frydenberg y Lewis, 2000).

En una investigación llevada a cabo por Figueroa et al. (2005) se encontraron diferencias significativas en el uso de estrategias de afrontamiento entre varones y mujeres en un grupo de estudiantes de nivel socioeconómico bajo de la provincia de Tucumán, Argentina. Las estrategias de afrontamiento que distinguen a las mujeres son: hacerse ilusiones, buscar apoyo social, buscar apoyo espiritual, autoinculparse, la estrategia de falta de afrontamiento y reducción de la tensión. Por otra parte, las estrategias que caracterizan a los varones son: distracción física e ignorar el problema.

En el estudio llevado a cabo por Casullo y Fernández Liporace (2001), se encontraron diferencias estadísticamente significativas en el tipo de estrategias de afrontamiento empleadas según el género de los estudiantes de la zona de Buenos Aires, Argentina. Las mujeres de Buenos Aires buscan más apoyo social, se concentran más en resolver el problema, se preocupan, invierten en amigos íntimos, buscan grupos de pertenencia, no afrontan sus problemas y se fijan en el lado positivo de la situación en mayor medida que los varones de la misma zona. Por otro lado, los varones emplean más que las mujeres las estrategias de ignorar el problema y buscar distracciones físicas. En este sentido, "el perfil de afrontamiento masculino es más homogéneo y el número de estrategias que emplean es más reducido" (Casullo y Fernández Liporace, 2001; 46).

En una investigación conducida por González Barrón et al. (2002) las estrategias de afrontamiento según género muestran que los varones destacan en el empleo de la distracción física, ignorar el problema y reservarlo para sí; mientras que las mujeres lo hacen en el estilo 
improductivo y en el de relación con los demás, a excepción de buscar ayuda profesional.

Por último, cabe destacar que Frydenberg y Lewis (1996b) encontraron que había diferencias significativas entre varones y mujeres en lo que respecta a la declaración de no tener ninguna preocupación; los varones tienden a manifestar en mayor medida que no tienen preocupaciones. Esto podría ser interpretado como que las mujeres sufren de más estrés que los varones o que estos últimos tienden más a negar sus preocupaciones.

Reconocemos que en la institución universitaria, el joven no sólo adquirirá los conocimientos teóricos y las experiencias prácticas relacionados con el ámbito del saber que se propuso profundizar sino que además hallará nuevas oportunidades de experimentar y desarrollar diversas competencias y habilidades que serán parte de su identidad adulta. A este respecto, Papalia y Wendkos (1999) señalan que en la universidad los estudiantes tienen la oportunidad de cuestionar supuestos desarrollados durante la infancia y, de este modo, construir una nueva identidad como adultos. Según estas autoras, los desafíos sociales y académicos de la universidad conducen a un crecimiento intelectual y moral. En este sentido, creemos sería beneficioso que las instituciones universitarias ofrecieran programas orientados a promover la adquisición de aprendizajes vinculados con un afrontamiento eficaz y productivo para lo cual se hace imprescindible conocer, en primer término, cómo están afrontando los estudiantes sus preocupaciones.

El propósito de este trabajo es estudiar el repertorio de estrategias de afrontamiento al estrés que utilizan los estudiantes universitarios de primer año y sus diferencias según la carrera que cursan y la variable sexo.

\section{MÉTODO}

El diseño de la presente investigación es exploratorio, descriptivo comparativo. Por un lado, nos disponemos a medir y describir los fenómenos del afrontamiento en estudiantes de Derecho, Educación Inicial, Ingeniería, Odontología y Psicología de primer año universitario. Por otro lado, pretendemos comparar las estrategias de afrontamiento y la carrera elegida, así como las estrategias de afrontamiento y el sexo de los estudiantes de dicho contexto. Recabamos los datos durante el segundo semestre del año 2007 a partir de una muestra en la que participaron jóvenes universitarios de cinco carreras distintas.

Realizamos el procesamiento de los datos a través del programa estadístico SPSS 14.0 para Windows.

\subsection{Sujetos}

El universo utilizado para la investigación, fue el conjunto de los estudiantes que cursan el primer año de las carreras de grado en Derecho, Educación Inicial, Ingeniería, Odontología y Psicología. Se realizaron las tramitaciones del caso para las autorizaciones institucionales y los correspondientes consentimientos, además de la aclaración a los jóvenes de su total libertad para elegir participar o no y que su opción no ocasionaría perjuicio alguno. La administración tuvo una duración de una media de 20 minutos y fue de tipo colectivo. La muestra total quedó conformada por 139 estudiantes universitarios de las carreras señaladas, con los porcentajes por carrera -sobre el universo- que se discriminan en la Tabla 1. Como datos descriptivos en Derecho el $71.42 \%$ eran mujeres y el $28.57 \%$ varones, el $78.57 \%$ no trabajaban, el $7.14 \%$ trabajaban y el $14.28 \%$ no respondieron, la media respecto a la edad era de 18, 67 años; en Educación Inicial

Tabla 1. Porcentaje de sujetos que completaron la escala, según carrera

\begin{tabular}{|c|c|}
\hline CARRERA & $\begin{array}{c}\text { \% DE ENCUESTADOS } \\
\text { DEL TOTAL }\end{array}$ \\
\hline Derecho & $70 \%$ \\
\hline Educación Inicial & $66.66 \%$ \\
\hline Ingeniería & $94.73 \%$ \\
\hline Odontología & $57.14 \%$ \\
\hline Psicología & $66.25 \%$ \\
\hline
\end{tabular}


el $90 \%$ eran mujeres y el $10 \%$ varones, el $60 \%$ no trabajaban y el $40 \%$ trabajaban, la media respecto a la edad era de 20 años; en Ingeniería el 13.88 eran mujeres y el $86.11 \%$ varones, el $69.44 \%$ no trabajaban, el $27.11 \%$ trabajaban y el $2.77 \%$ no respondieron, la media respecto a la edad era de 20.06 años; en Odontología el $75 \%$ eran mujeres y el $25 \%$ varones, el $100 \%$ no trabajaban, la media respecto a la edad era de 18,58 años; en Psicología el 84.9\% eran mujeres, el $13.2 \%$ varones y el $1.88 \%$ no respondieron, el $67.92 \%$ no trabajaban, el $26.41 \%$ trabajaban y el $5.66 \%$ no respondieron, la media respecto a la edad era de 20.28 años.

\subsection{Instrumento}

Se administró la adaptación al Uruguay de la Escala de Afrontamiento para Adolescentes (ACS) en su forma específica (García Pérez, 2008). El ACS es una escala elaborada por Frydenberg y Lewis en 1993 y traducida al español por Pereña y Seisdedos en 1996 cuyo objetivo es la evaluación de las estrategias de afrontamiento.

El ACS es un inventario de autoinforme compuesto de 80 elementos, 79 de tipo cerrado y uno final abierto, agrupados en 18 factores diferentes, cada uno de los cuales se compone de entre 3 y 5 elementos y reflejan 18 estrategias de afrontamiento, que se han identificado conceptual y empíricamente.
Cada uno de los 79 elementos cerrados describe una conducta de afrontamiento específica y se puntúa mediante una escala de tipo Likert de cinco puntos: 1 . No me ocurre nunca o no lo hago; 2 . Me ocurre o lo hago raras veces; 3 . Me ocurre o lo hago algunas veces; 4. Me ocurre o lo hago a menudo; 5. Me ocurre o lo hago con mucha frecuencia (Frydenberg y Lewis, 1996a).

La escala presenta dos formas de aplicación: una general y una específica. La forma general del instrumento indaga cómo el sujeto afronta sus problemas en general ya que se ha observado que las estrategias de afrontamiento empleadas con mayor frecuencia por una persona tienden a ser relativamente estables independientemente de las características del problema. Por otra parte, la forma específica permite evaluar las respuestas a un problema particular citado por el sujeto o propuesto por el examinador. La administración puede ser individual o colectiva, tomando como tiempo total de aplicación 15 minutos aproximadamente. Sin embargo, no hay límite de tiempo para completarla.

\section{RESULTADOS}

En la tabla 2 exponemos los resultados de cada una de las 18 estrategias de afrontamiento al estrés que obtuvieron los sujetos de investigación según la carrera que cursan (describimos

Tabla 2. Descripción de los perfiles de estrategias de afrontamiento según carrera

\begin{tabular}{|c|c|c|c|c|c|c|c|c|c|c|c|c|c|c|c|}
\hline \multirow{3}{*}{ Estrategias de Afrontamiento } & \multicolumn{15}{|c|}{ Carrera } \\
\hline & \multicolumn{3}{|c|}{ Derecho } & \multicolumn{3}{|c|}{$\begin{array}{c}\text { Educación } \\
\text { Inicial }\end{array}$} & \multicolumn{3}{|c|}{ Ingeniería } & \multicolumn{3}{|c|}{ Odontologia } & \multicolumn{3}{|c|}{ Psicologia } \\
\hline & $\mathrm{N}$ & $M$ & DS & $\mathbf{N}$ & M & DS & $\mathbf{N}$ & M & DS & $\mathbf{N}$ & M & DS & $\mathbf{N}$ & M & DS \\
\hline Buscar apoyo social (As) & 28 & 68.85 & 13.61 & 10 & 77.2 & 15.09 & 36 & 60.66 & 16.38 & 12 & 60 & 12.76 & 53 & 69.66 & 17.94 \\
\hline $\begin{array}{c}\text { Concentrarse en resolver el } \\
\text { problema }(R p)\end{array}$ & 28 & 73.14 & 13.85 & 10 & 73.2 & 15.32 & 36 & 68.55 & 16.17 & 12 & 74.33 & 11.99 & 53 & 74.94 & 11.34 \\
\hline Esforzarse y tener éxito (Es) & 28 & 71.28 & 11.82 & 10 & 71.6 & 11.53 & 36 & 70.88 & 12.67 & 12 & 77.33 & 12.45 & 53 & 70.41 & 13.22 \\
\hline Preocuparse (Pr) & 28 & 79.71 & 12.36 & 10 & 78.4 & 12.39 & 36 & 72.88 & 13.72 & 12 & 75.66 & 7.32 & 53 & 75.39 & 10.53 \\
\hline Invertir en amigos intimos (Ai) & 28 & 74 & 12 & 10 & 75.6 & 16.16 & 36 & 61.88 & 12.55 & 12 & 72.33 & 17.92 & 53 & 64.9 & 16.92 \\
\hline Buscar pertenencia $(\mathrm{Pe})$ & 28 & 72.85 & 12.33 & 10 & 72 & 12.5 & 36 & 66.11 & 10.45 & 12 & 74.33 & 14.71 & 53 & 68.52 & 14.73 \\
\hline Hacerse ilusiones $(\mathrm{Hi})$ & 28 & $c 0$ & 15.92 & 10 & 60 & 11.62 & 36 & 58.77 & 15.82 & 12 & 62.66 & 15.66 & 53 & 56.9 & 15.28 \\
\hline $\begin{array}{l}\text { Estrategia de falta de } \\
\text { afrontamiento }(\mathrm{Na})\end{array}$ & 28 & 42.14 & 18.03 & 10 & 42.8 & 11.93 & 36 & 42.33 & 14.63 & 12 & 38.66 & 12.91 & 53 & 42.71 & 14.6 \\
\hline Reducción de la tensión (Rt) & 28 & 43.85 & 16.05 & 10 & 47.2 & 14.94 & 36 & 45.55 & 17 & 12 & 38.33 & 7.9 & 53 & 45.05 & 13.9 \\
\hline Acción social (So) & 28 & 33.03 & 9.16 & 10 & 36 & 16.12 & 36 & 38.05 & 14.84 & 12 & 32.08 & 4.5 & 53 & 37.54 & 10.63 \\
\hline Ignorar el problema (Ip) & 28 & 38.39 & 15.39 & 10 & 44 & 13.49 & 36 & 45.13 & 18.14 & 12 & 37.5 & 15.44 & 53 & 37.64 & 12.61 \\
\hline Autoinculparse $(\mathrm{Cu})$ & 28 & 60.35 & 22.44 & 10 & 59 & 17.6 & 36 & 57.5 & 16.92 & 12 & 51.66 & 8.61 & 53 & 61.88 & 17.15 \\
\hline Reservarlo para si (Re) & 28 & 52.14 & 21.74 & 10 & 51.5 & 15.82 & 36 & 57.63 & 19.17 & 12 & 49.58 & 8.9 & 53 & 51.41 & 17.46 \\
\hline Buscar apoyo espiritual (Ae) & 28 & 43.21 & 13.06 & 10 & 53.5 & 22.24 & 36 & 44.3 & 15.86 & 12 & 45.83 & 14.59 & 53 & 41.98 & 15.51 \\
\hline Fijarse en lo positivo $(\mathrm{Po})$ & 28 & 66.25 & 15.43 & 10 & 71.5 & 17.95 & 36 & 62.91 & 13.69 & 12 & 74.58 & 10.1 & 53 & 65.75 & 12.91 \\
\hline Buscar ayuda profesional (Ap) & 28 & 48.75 & 17.93 & 10 & 51 & 15.77 & 36 & 50.41 & 16.91 & 12 & 47.08 & 14.53 & 53 & 57.16 & 18.43 \\
\hline Buscar diversiones relajantes (Dr) & 28 & 81.75 & 11.75 & 10 & 79.8 & 23.14 & 36 & 73.3 & 18.59 & 12 & 72.33 & 15.02 & 53 & 76.73 & 16.58 \\
\hline Distracción fisica (Fi) & 28 & 60.25 & 25.93 & 10 & 55.3 & 23.43 & 36 & 68.25 & 24.15 & 12 & 47.25 & 17.43 & 53 & 54.67 & 25.5 \\
\hline
\end{tabular}


el número de estudiantes en análisis para cada carrera, la media de las puntuaciones ajustadas que obtuvieron en cada factor y el desvío estándar correspondiente). Luego, graficamos los perfiles de afrontamiento obtenidos por estos estudiantes de acuerdo con el mismo criterio (Gráfico 1).

\subsection{Comparación estadística de las medias de los factores de afrontamiento entre carreras}

El análisis de asimetría dio que los factores As, Rp, Es, Pr, Ai, Pe, Hi, Na, Rt, Ip, Cu, Re, Po, Ap, Dr, Fi presentan una distribución normal, en tanto que los factores So y Ae no presentan tal distribución. En consecuencia, decidimos utilizar la prueba paramétrica ANOVA de comparación de medias para analizar el primer grupo de factores (tabla 3, página siguiente), mientras que usamos la prueba no paramétrica $\mathrm{H}$ de KruskalWallis para estudiar el segundo grupo de factores (tabla 5, página 170).

Considerando las medias de los factores entre las carreras, observamos diferencias significativas en los factores As y Ai (tabla 3). Con respecto al factor As, el estadístico Anova es de 3.391, con 4 grados de libertad y un nivel crítico de $0.011(p<0.05)$, $y$, en cuanto al factor $\mathrm{Ai}$, el estadístico Anova es de 4.002, con 4 grados de libertad y un nivel crítico de $0.004(p<0.05)$.

Con el objetivo de determinar específicamente entre qué carreras se hallan las diferencias estadísticamente significativas observadas en los factores As y Ai (tabla 3) se ejecutó un Análisis Post Hoc Tukey (tabla 4, página siguiente).

Se encontraron diferencias estadísticamente significativas entre las carreras Ingeniería y Educación Inicial para el factor As y entre las carreras Derecho e Ingeniería para el factor Ai (tabla 4). Notamos que existen diferencias significativas entre los estudiantes de Ingeniería y Educación Inicial en la estrategia de afrontamiento: "Buscar apoyo social" $(0.039 ; p<0.05)$, estrategia puntuada más alto por los estudiantes de Educación Inicial $(M=77.2 ; D S=15.09)$ que por los estudiantes de Ingeniería ( $M=60.66$; $D S=16.38$ ). Asimismo, hallamos diferencias significativas entre los estudiantes de Derecho y los estudiantes de Ingeniería en la estrategia de afrontamiento "Invertir en amigos íntimos" (0.014; $p<0.05)$, estrategia puntuada más alto por los estudiantes de Derecho $(\mathrm{M}=74 ; \mathrm{DS}=12)$ que por los estudiantes de Ingeniería ( $M=61.88 ; \mathrm{DS}=12.55)$.

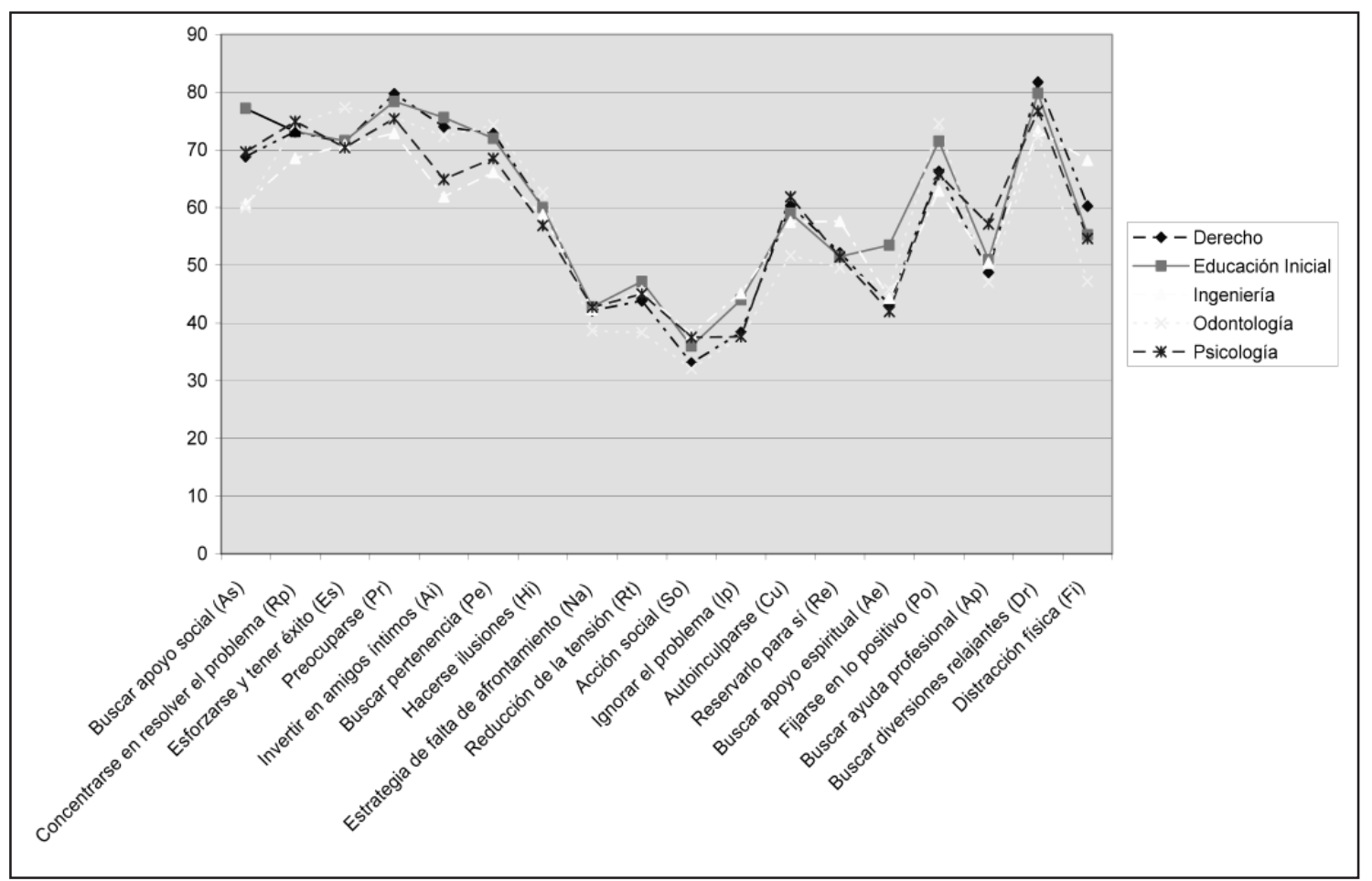

Gráfico 1. Perfiles de estrategias de afrontamiento por carrera 
Tabla 3. ANOVA: Comparación de las medias de los factores As, Rp, Es, Pr, Ai, Pe, Hi, Na, Rt, Ip, Cu, Re, Po, Ap, Dr, Fi según carrera

\begin{tabular}{|c|c|c|c|c|c|}
\hline & Suma de cuadrados & gl & Media cuadrática & $\mathrm{F}$ & Sig. \\
\hline $\begin{array}{c}\text { Buscar apoyo social (As) } \\
\text { Inter-grupos } \\
\text { Intra-grupos } \\
\text { Total }\end{array}$ & $\begin{array}{c}3542.005 \\
34986.915 \\
38528.921\end{array}$ & $\begin{array}{c}4 \\
134 \\
138 \\
\end{array}$ & $\begin{array}{l}885.501 \\
261.096\end{array}$ & 3.391 & $0.011^{*}$ \\
\hline $\begin{array}{c}\text { Concentrarse en resolver el problema (Rp) } \\
\text { Inter-grupos } \\
\text { Intra-grupos } \\
\text { Total }\end{array}$ & $\begin{array}{c}924.773 \\
24725.414 \\
25650.187\end{array}$ & $\begin{array}{c}4 \\
134 \\
138 \\
\end{array}$ & $\begin{array}{l}231.193 \\
184.518\end{array}$ & 1.253 & 0.292 \\
\hline $\begin{array}{c}\text { Esforzarse y tener éxito (Es) } \\
\text { Inter-grupos } \\
\text { Intra-grupos } \\
\text { Total }\end{array}$ & $\begin{array}{c}484.033 \\
21401.204 \\
21885.237\end{array}$ & $\begin{array}{c}4 \\
134 \\
138 \\
\end{array}$ & $\begin{array}{l}121.008 \\
159.710\end{array}$ & 0.758 & 0.555 \\
\hline $\begin{array}{l}\text { Preocuparse }(\mathrm{Pr}) \\
\text { Inter-grupos } \\
\text { Intra-grupos } \\
\text { Total } \\
\end{array}$ & $\begin{array}{c}810.107 \\
18467.016 \\
19277.122\end{array}$ & $\begin{array}{c}4 \\
134 \\
138 \\
\end{array}$ & $\begin{array}{l}202.527 \\
137.814\end{array}$ & 1.470 & 0.215 \\
\hline $\begin{array}{c}\text { Invertir en amigos intimos (Ai) } \\
\text { Inter-grupos } \\
\text { Intra-grupos } \\
\text { Total } \\
\end{array}$ & $\begin{array}{c}3607.137 \\
30193.151 \\
33800.288\end{array}$ & $\begin{array}{c}4 \\
134 \\
138 \\
\end{array}$ & $\begin{array}{l}901.784 \\
225.322\end{array}$ & 4.002 & $0.004^{*}$ \\
\hline $\begin{array}{c}\text { Buscar pertenencia }(\mathrm{Pe}) \\
\text { Inter-grupos } \\
\text { Intra-grupos } \\
\text { Total } \\
\end{array}$ & $\begin{array}{c}1121.804 \\
23018.858 \\
24140.662 \\
\end{array}$ & $\begin{array}{c}4 \\
134 \\
138 \\
\end{array}$ & $\begin{array}{l}280.451 \\
171.783\end{array}$ & 1.633 & 0.170 \\
\hline $\begin{array}{c}\text { Hacerse ilusiones }(\mathrm{Hi}) \\
\text { Inter-grupos } \\
\text { Intra-grupos } \\
\text { Total } \\
\end{array}$ & $\begin{array}{c}423.734 \\
31673.417 \\
32097.151\end{array}$ & $\begin{array}{c}4 \\
134 \\
138 \\
\end{array}$ & $\begin{array}{l}105.933 \\
236.369\end{array}$ & 0.448 & 0.774 \\
\hline $\begin{array}{c}\text { La estrategia de falta de afrontamiento }(\mathrm{Na}) \\
\text { Inter-grupos } \\
\text { Intra-grupos } \\
\text { Total }\end{array}$ & $\begin{array}{c}168.068 \\
30496.450 \\
30664.518\end{array}$ & $\begin{array}{c}4 \\
134 \\
138 \\
\end{array}$ & $\begin{array}{c}42.017 \\
227.585\end{array}$ & 0.185 & 0.946 \\
\hline $\begin{array}{l}\text { Reducción de la tensión (Rt) } \\
\text { Inter-grupos } \\
\text { Intra-grupos } \\
\text { Total } \\
\end{array}$ & $\begin{array}{c}597.291 \\
29829.414 \\
30426.705\end{array}$ & $\begin{array}{c}4 \\
134 \\
138\end{array}$ & $\begin{array}{l}149.323 \\
222.608\end{array}$ & 0.671 & 0.613 \\
\hline $\begin{array}{l}\text { Ignorar el problema }(\mathrm{Ip}) \\
\text { Inter-grupos } \\
\text { Intra-grupos } \\
\text { Total } \\
\end{array}$ & $\begin{array}{c}1548.331 \\
30472.173 \\
32020.504\end{array}$ & $\begin{array}{c}4 \\
134 \\
138 \\
\end{array}$ & $\begin{array}{l}382.083 \\
227.404\end{array}$ & 1.702 & 0.153 \\
\hline $\begin{array}{c}\text { Autoinculparse }(\mathrm{Cu}) \\
\text { Inter-grupos } \\
\text { Intra-grupos } \\
\text { Total } \\
\end{array}$ & $\begin{array}{c}1202.311 \\
42539.416 \\
43741.727 \\
\end{array}$ & $\begin{array}{c}4 \\
134 \\
138 \\
\end{array}$ & $\begin{array}{l}300.578 \\
317.458\end{array}$ & 0.947 & 0.439 \\
\hline $\begin{array}{l}\text { Reservarlo para si (Re) } \\
\text { Inter-grupos } \\
\text { Intra-grupos } \\
\text { Total }\end{array}$ & $\begin{array}{c}1090.917 \\
44640.019 \\
45730.935\end{array}$ & $\begin{array}{c}4 \\
134 \\
138 \\
\end{array}$ & $\begin{array}{l}272.729 \\
333.134\end{array}$ & 0.819 & 0.515 \\
\hline $\begin{array}{l}\text { Fijarse en lo positivo (Po) } \\
\text { Inter-grupos } \\
\text { Intra-grupos } \\
\text { Total }\end{array}$ & $\begin{array}{c}1521.678 \\
25695.228 \\
27216.906\end{array}$ & $\begin{array}{c}4 \\
134 \\
138 \\
\end{array}$ & $\begin{array}{l}380.420 \\
191.755\end{array}$ & 1.984 & 0.101 \\
\hline $\begin{array}{c}\text { Buscar ayuda profesional (Ap) } \\
\text { Inter-grupos } \\
\text { Intra-grupos } \\
\text { Total }\end{array}$ & $\begin{array}{c}2079.238 \\
40938.388 \\
43017.626\end{array}$ & $\begin{array}{c}4 \\
134 \\
138\end{array}$ & $\begin{array}{l}519.809 \\
305.510\end{array}$ & 1.701 & 0.153 \\
\hline $\begin{array}{c}\text { Buscar diversiones relajantes (Dr) } \\
\text { Inter-grupos } \\
\text { Intra-grupos } \\
\text { Total } \\
\end{array}$ & $\begin{array}{c}1453.852 \\
37439.457 \\
38893.309\end{array}$ & $\begin{array}{c}4 \\
134 \\
138 \\
\end{array}$ & $\begin{array}{l}363.463 \\
279.399\end{array}$ & 1.301 & 0.273 \\
\hline $\begin{array}{l}\text { Distracción fisica (Fi) } \\
\text { Inter-grupos } \\
\text { Intra-grupos } \\
\text { Total } \\
\end{array}$ & $\begin{array}{c}6.226 \\
98.364 \\
104.59 \\
\end{array}$ & $\begin{array}{c}4 \\
134 \\
138 \\
\end{array}$ & $\begin{array}{l}1.556 \\
0.734\end{array}$ & 2.120 & 0.082 \\
\hline
\end{tabular}

Tabla 4. Análisis Post Hoc Tukey: As y Ai según carrera

\begin{tabular}{|c|c|c|c|}
\hline & Carreras & $\begin{array}{c}\text { Diferencia de } \\
\text { Medias }\end{array}$ & Sig \\
\hline \multirow{10}{*}{$\begin{array}{c}\text { Buscar apoyo } \\
\text { social (As) }\end{array}$} & Derecho-Psicologia & -080323 & 1.000 \\
\hline & Derecho-Ingeniería & 8.19048 & 0.266 \\
\hline & Derecho-Odontologia & 8.85714 & 0.507 \\
\hline & Derecho-Educación Inicial & -8.34286 & 0.628 \\
\hline & Psicologia-Ingenieria & 8.99371 & 0.080 \\
\hline & Psicologia-Odontologia & 9.66038 & 0.339 \\
\hline & Psicologia-Educación Inicial & -7.53962 & 0.658 \\
\hline & Ingenieria-Odontologia & 0.66667 & 1.000 \\
\hline & Ingenieria-Educación Inicial & -16.53333 & $0.039^{*}$ \\
\hline & Odontologia-Educación Inicial & -17.20000 & 0.100 \\
\hline \multirow{11}{*}{$\begin{array}{c}\text { Invertir en } \\
\text { amigos intimos } \\
\text { (Ai) }\end{array}$} & & & \\
\hline & & 909434 & 0.077 \\
\hline & $\begin{array}{l}\text { Derecho-Psicologia } \\
\text { Derecho-Ingeniería }\end{array}$ & 12.11111 & $0.014^{\circ}$ \\
\hline & Derecho-Odontologia & 1.66667 & 0.998 \\
\hline & Derecho-Educación Inicial & -1.60000 & 0.998 \\
\hline & Psicologia-Ingenieria & 3.01677 & 0.885 \\
\hline & Psicologia-Odontologia & -7.42767 & 0.535 \\
\hline & Psicologia-Educación Inicial & -10.69434 & 0.241 \\
\hline & Ingenieria-Odontologia & -10.44444 & 0.232 \\
\hline & Ingenieria-Educación Inicial & -13.71111 & 0.085 \\
\hline & Odontologia-Educación Inicial & -3.26667 & 0.986 \\
\hline
\end{tabular}


Como se puede observar en la tabla 5, no se hallaron diferencias estadísticamente significativas en los factores So ni Ae entre los grupos definidos según carrera.

Tabla 5. K muestras independientes: H de Kruskal-Wallis: Comparación de medias de los factores So y Ae según carrera

\begin{tabular}{|c|c|c|c|}
\hline & Chi-cuadrado & gl & Sig. asintót. \\
\hline Acción Social (So) & 3.829 & 3 & 0.281 \\
\hline Buscar apoyo espiritual (Ae) & 1.514 & 3 & 0.679 \\
\hline
\end{tabular}

\subsection{Descripción de los perfiles de estrate- gias de afrontamiento según sexo}

En la tabla 6 exponemos los resultados de cada una de las 18 estrategias de afrontamiento al estrés que obtuvieron los sujetos de investigación según su sexo (describimos el número de estudiantes en análisis según sexo, la media de las puntuaciones ajustadas que obtuvieron en cada factor y el desvío estándar correspondiente). Luego, graficamos los perfiles de afrontamiento obtenidos por estos estudiantes de acuerdo con el mismo criterio (Gráfico 2, página siguiente).

\subsection{Comparación estadística de las medias de los factores de afrontamiento según sexo}

Para analizar todos los factores según el sexo de los sujetos recurrimos a la prueba no paramétri- ca $U$ de Mann-Whitney (tabla 7, página siguiente).

Considerando las medias de todos los factores entre los sexos, observamos diferencias significativas en los factores As, Pr, Pe y Dr (tabla 7). En el factor As el estadístico $U$ de Mann-Whitney es de 1291 con un nivel crítico bilateral de $0.000(p<0.05)$, en el factor Pr el estadístico $U$ de Mann-Whitney es de 1585 con un nivel crítico bilateral de $0.005(p<0.05)$, en el factor Pe el estadístico $U$ de Mann-Whitney es de 1567 con un nivel crítico bilateral de 0.004 $(p<0.05), y$, en el factor $D r$ el estadístico $U$ de Mann-Whitney es de 1625 con un nivel crítico bilateral de $0.008(p<0.05)$. Notamos que existen diferencias significativas de acuerdo al sexo en las estrategias de afrontamiento: "Buscar apoyo social" $(0.000 ; p<0.05)$, estrategia puntuada más alto por las mujeres $(\mathrm{M}=71.21 ; \mathrm{DS}=15.93)$ que por los varones $(M=59.37$; $D S=15.58)$; "Preocuparse" (0.005; $p<0.05)$, estrategia puntuada más alto por las mujeres $(\mathrm{M}=78.11 ; \mathrm{DS}=9.79)$ que por los varones ( $M=71.84$; $D S=13.92)$; "Buscar pertenencia", estrategia puntuada más alto por las mujeres ( $M=71.95 ; D S=12.72)$ que por los varones (M=65.41; $\mathrm{DS}=13.28) ; \mathrm{y}$, "Buscar diversiones relajantes", estrategia puntuada más alto por las mujeres $(\mathrm{M}=79.57$; $\mathrm{DS}=16.74)$ que por los varones $(\mathrm{M}=72.33$; $\mathrm{DS}=15.69)$.

Tabla 6. Descripción de los perfiles de estrategias de afrontamiento según sexo

\begin{tabular}{|c|c|c|c|c|c|c|}
\hline \multirow{2}{*}{ Estrategias de Afrontamiento } & \multicolumn{5}{|c|}{ Sarones } \\
\cline { 2 - 7 } & \multicolumn{3}{|c|}{ Mujeres } & \multicolumn{3}{c|}{ D } \\
\cline { 2 - 7 } & N & M & DS & N & M & DS \\
\hline Buscar apoyo social (As) & 87 & 71.21 & 15.93 & 51 & 59.37 & 15.58 \\
\hline Concentrarse en resolver el problema (Rp) & 87 & 74.34 & 12.55 & 51 & 70.03 & 15.16 \\
\hline Esforzarse y tener éxito (Es) & 87 & 72.22 & 12.84 & 51 & 69.88 & 12.24 \\
\hline Preocuparse (Pr) & 87 & 78.11 & 9.79 & 51 & 71.84 & 13.92 \\
\hline Invertir en amigos intimos (Ai) & 87 & 69.01 & 15.57 & 51 & 64.54 & 15.67 \\
\hline Buscar pertenencia (Pe) & 87 & 71.95 & 12.72 & 51 & 65.41 & 13.28 \\
\hline Hacerse ilusiones (Hi) & 87 & 60.04 & 14.4 & 51 & 56.86 & 16.47 \\
\hline Estrategia de falta de afrontamiento (Na) & 87 & 43.54 & 14.64 & 51 & 40 & 15.3 \\
\hline Reducción de la tensión (Rt) & 87 & 45.47 & 13.95 & 51 & 42.98 & 16.41 \\
\hline Acción social (So) & 87 & 35.91 & 10.82 & 51 & 36.76 & 13.4 \\
\hline Ignorar el problema (Ip) & 87 & 39.48 & 15.25 & 51 & 41.76 & 15.12 \\
\hline Autoinculparse (Cu) & 87 & 61.32 & 18.87 & 51 & 55.78 & 15.5 \\
\hline Reservarlo para si (Re) & 87 & 51.78 & 17.78 & 51 & 55.39 & 18.94 \\
\hline Buscar apoyo espiritual (Ae) & 87 & 44.82 & 15.02 & 51 & 42.74 & 16.89 \\
\hline Fijarse en lo positivo (Po) & 87 & 67.52 & 14.93 & 51 & 64.41 & 12.31 \\
\hline Buscar ayuda profesional (Ap) & 87 & 54.08 & 18.08 & 51 & 49.6 & 16.87 \\
\hline Buscar diversiones relajantes (Dr) & 87 & 79.57 & 16.74 & 51 & 72.33 & 15.69 \\
\hline Distracción física (Fi) & 87 & 56.4 & 25.49 & 51 & 62.58 & 24.26 \\
\hline
\end{tabular}




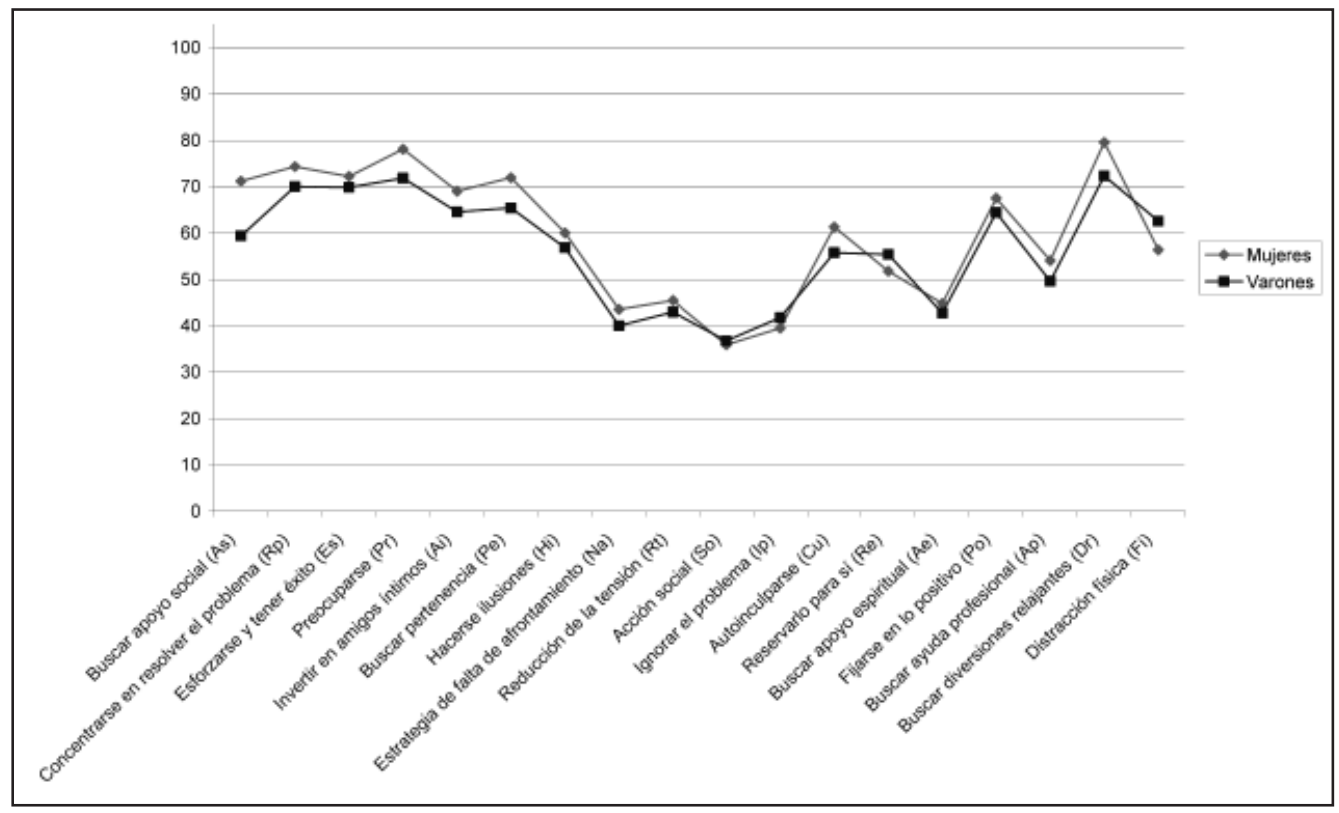

Gráfico 2. Perfiles de estrategias de afrontamiento por sexo

Tabla 7. Dos muestras independientes: U de Mann-Whitney: Comparación de medias de los factores de afrontamiento según sexo

\begin{tabular}{|c|c|c|c|c|}
\hline & U de Mann-Whitney & W de Wilcoxon & Z & Sig. asintót. (bilateral) \\
\hline Buscar apoyo social (As) & 1291.000 & 2617.000 & -4.104 & $0.000^{*}$ \\
\hline Concentrarse en resolver el problema (Rp) & 1825.500 & 3151.500 & -1.742 & 0.082 \\
\hline Esforzarse y tener éxito (Es) & 1960.000 & 3286.000 & -1.146 & 0.252 \\
\hline Preocuparse (Pr) & 1585.000 & 2911.000 & -2.816 & $0.005^{\star}$ \\
\hline Invertir en amigos intimos (Ai) & 1840.000 & 3166.000 & -1.676 & 0.094 \\
\hline Buscar pertenencia (Pe) & 1567.000 & 2893.000 & -2.886 & $0.004^{*}$ \\
\hline Hacerse ilusiones (Hi) & 1891.000 & 3217.000 & -1.450 & 0.147 \\
\hline Estrategia de falta de afrontamiento (Na) & 1852.000 & 3178.000 & -1.624 & 0.104 \\
\hline Reducción de la tensión (Rt) & 1883.500 & 3209.500 & -1.483 & 0.138 \\
\hline Acción social (So) & 2170.500 & 3496.500 & -0.215 & 0.830 \\
\hline Ignorar el problema (Ip) & 2019.500 & 5847.500 & -0.883 & 0.377 \\
\hline Autoinculparse (Cu) & 1813.500 & 3139.500 & -1.794 & 0.073 \\
\hline Reservarlo para si (Re) & 1938.500 & 5766.500 & -1.240 & 0.215 \\
\hline Buscar apoyo espiritual (Ae) & 1939.000 & 3265.000 & -1.244 & 0.214 \\
\hline Fijarse en lo positivo (Po) & 1963.500 & 3289.500 & -1.133 & 0.257 \\
\hline Buscar ayuda profesional (Ap) & 1858.500 & 3184.500 & -1.596 & 0.111 \\
\hline Buscar diversiones relajantes (Dr) & 1625.000 & 2951.000 & -2.641 & $0.008^{\star}$ \\
\hline Distracción fisica (Fi) & 1847.000 & 5675.000 & -1.646 & 0.100 \\
\hline
\end{tabular}

\section{DISCUSIÓN}

Con el fin de dar respuesta a nuestro interés de estudiar cómo afrontan sus preocupaciones los estudiantes que cursan distintas carreras universitarias, llevamos a cabo una investigación de tipo exploratoria descriptiva comparativa en la cual administramos la escala ACS en su forma específica a 139 estudiantes de Derecho, Educación Inicial, Ingeniería, Odontología y Psicología de primer año universitario.

Con respecto a la interrogante que nos planteamos de si existen diferencias significativas en el uso de estrategias de afrontamiento al estrés entre los estudiantes de Derecho, Educación Inicial, Ingeniería, Odontología y Psicología 
de primer año, se hallaron diferencias con significancia estadística entre los estudiantes de Derecho e Ingeniería en la estrategia de afrontamiento "invertir en amigos íntimos" y entre los estudiantes de Ingeniería y Educación Inicial en la estrategia de afrontamiento "buscar apoyo social". Los estudiantes de Derecho buscan comprometerse en alguna relación personal de tipo íntimo como forma de afrontar sus preocupaciones más frecuentemente que los estudiantes de Ingeniería y los estudiantes de Educación Inicial tienden a compartir sus problemas con los demás y buscar apoyo en su resolución con mayor frecuencia que sus pares de Ingeniería.

Por otra parte, a raíz del estudio de la comparación entre las variables sexo y estrategias de afrontamiento, se hallaron diferencias estadísticamente significativas en el uso de estrategias de afrontamiento entre las mujeres y los varones de la muestra. Las mujeres reportaron recurrir con mayor frecuencia que los varones a "buscar apoyo social", o sea, compartir el problema con otros y buscar apoyo en su resolución, "buscar pertenencia", o sea, enfocarse en sus relaciones con los demás en general y, más en concreto, preocuparse por lo que los demás piensan de ellas, "preocuparse", es decir, expresar temor por el futuro en términos generales o, más específicamente, preocupación por la felicidad futura, y "buscar diversiones relajantes", es decir, recurrir a actividades de ocio y relajantes a la hora de afrontar sus preocupaciones. Estos hallazgos coinciden con el planteo de Frydenberg y Lewis (1991) y lo reportado en diversas investigaciones (Casullo y Fernández Liporace, 2001; Figueroa et al., 2005; González Barrón et al., 2002) de que, frente a una dificultad, las mujeres buscan más apoyo social y tienden a focalizarse en las relaciones más que los varones, pues aceptan más la comprensión y el apoyo y suelen compartir con otros lo que les sucede en mayor medida que ellos.

\section{CONCLUSIONES}

Considerando lo manifestado por Frydenberg y Lewis (1996a), estos resultados podrían deberse a que personas que utilizan en mayor medida estrategias de afrontamiento vinculadas con la búsqueda de ayuda, apoyo o interacción con otros tienden a elegir carreras que implican contacto personal mientras que los jóvenes que optan por carreras científicas donde se enfatiza más la racionalidad individual emplean estas estrategias con menor frecuencia.

A modo de sugerencia, a partir del presente estudio, nos planteamos otros objetivos de interés como: efectuar una comparación entre estudiantes de la misma carrera pero de grados distintos (por ejemplo, el primer y último año de la misma carrera) ya que tal vez el tipo de formación recibida moldee los comportamientos de afrontamiento en base a las competencias requeridas en la misma; explorar los estilos de afrontamiento que emplean los estudiantes universitarios; correlacionar el afrontamiento con diversas variables que han sido asociadas en otras poblaciones como: el bienestar psicológico, el optimismo y el pesimismo, la autoeficacia y el rendimiento académico.

Consideramos que es de suma relevancia que se preste atención a lo que les ocurre a los jóvenes universitarios, pues se encuentran en una etapa vital en la que se espera que tomen decisiones respecto a su proyecto de vida y al mismo tiempo se encuentran en transición ya que no son considerados adolescentes pero tampoco se sienten adultos, lo cual pueden vivir como muy estresante. La Universidad como institución educativa aporta conocimientos y experiencias que van más allá de lo curricular. En este sentido, la institución universitaria puede constituir un marco a partir del cual se les ayude a manejar sus problemas y preocupaciones de modo adaptativo y beneficioso tanto para sí mismos como para quienes les rodean; contribuyendo a formar personas más concientes y responsables de sus acciones y emociones frente a las dificultades y adversidades.

\section{REFERENCIAS}

Casullo, M., Fernández Liporace, M. (2001). Estrategias de afrontamiento en estudiantes adolescentes. Revista del Instituto de Investigaciones de la Facultad de Psicología/ UBA, 6 (1): 25-49.

Figueroa, M., Contini, N., Lacunza, A., Levín, M., Estévez Suedan, A. (2005). Las estrategias de afrontamiento y su relación con el nivel de bienestar psicológico. Un estudio con adolescentes de nivel socioeconómico bajo de Tucumán (Argentina). Anales de Psicología, 21(1): 66-72.

Frydenberg, E. (1997). Adolescent Coping. Theoretical and Research Perspectives. Nueva York: Routledge. 
Frydenberg, E. (2002). Beyond Coping: Some Paradigms to Consider en Frydenberg, E. (Comp.), Beyond Coping. Meeting goals, visions, and challenges. Nueva York: Oxford University Press.

Frydenberg, E., Lewis, R. (1991). Adolescent coping: the different ways in which boys and girls cope. Journal of Adolescence, 14, 119-133.

Frydenberg, E., Lewis, R. (1993). Boys play sport and girls turn to others: age, gender and ethnicity as determinants of coping. Journal of Adolescence, 16, 253-266.

Fydenberg, E., Lewis, R. (1996a). ACS. Escalas de Afrontamiento para Adolescentes. Manual. Madrid: TEA.

Frydenberg, E., Lewis, R. (1996b). The coping strategies used by adolescents in intact and in separated families. Australian Joural of Guidance \& Counselling, 6 (1): 87-97.

Frydenberg, E., Lewis, R. (2000). Teaching Coping to Adolescents: When and to Whom? American Educational Research Journal, 37 (3): 727-745.

Frydenberg, E., Rowley, G. (1998). Coping with social issues: What Australian university students do. Issues in Educational Research, 8 (1): 33- 48.
García Pérez, R. (2008) Estrategias y Estilos de Afrontamiento al Estrés en una muestra de adolescentes uruguayos. Adaptación y Baremación de la Escala de Afrontamiento al estrés para Adolescentes (ACS). Tesis Doctoral, Universidad de Murcia, Murcia, España.

González Barrón, R., Montoya Castilla, I., Casullo, M., Benabéu Verdú, J. (2002). Relación entre estilos y estrategias de afrontamiento y bienestar psicológico en adolescentes. Psicothema, 14 (2): 363-368.

Johnson, D., Johnson, R. (2002). Teaching Students How to Cope with Adversity: the Three Cs en Frydenberg, E. (Comp.), Beyond Coping. Meeting goals, visions, and challenges. Nueva York: Oxford University Press.

Lazarus, R. (1999). Stress and Emotion. A New Synthesis. Nueva York: Springer Publishing Company.

Lazarus, R., Lazarus, B. (1994). Passion \& reason. Nueva York: Oxford University Press.

Lazarus, R. y Folkman, S. (1987). Transactional Theory and research on emotion and coping. European Personality, 1, 141-169.

Papalia, D., Wendkos, O. (1999). Desarrollo Humano. México: McGraw-Hill. Sexta edición. 